\title{
Application of a three-dimensional high-definition exoscope in minimally invasive transforaminal lumbar interbody fusion
}

\section{Zhi-Peng Yao}

Southern Medical University

\section{Chen-Jie Xiong}

General hospital of Central Theater Command of PLA

\section{Bi-Wang Huang}

General hospital of Central Theater Command of PLA

Ya-Wei Yao

Southern Medical University

\section{Hui Kang}

General hospital of Central Theater Command of PLA

Feng Xu ( $\nabla$ fengxu1969@163.com)

General hospital of Central Theater Command of PLA

\section{Technical note}

Keywords: 3D, Exoscope, MIS-TLIF, Lumbar degenerative diseases

Posted Date: July 21st, 2020

DOl: https://doi.org/10.21203/rs.3.rs-41963/v1

License: (c) (i) This work is licensed under a Creative Commons Attribution 4.0 International License. Read Full License 


\section{Abstract \\ Background}

Minimally invasive transforaminal lumbar interbody fusion (MIS-TLIF) is becoming increasingly popular in the treatment of lumbar degenerative diseases. Microscope assisted MIS-TLIF was also reported favorable clinical outcomes and low complication rates, while limitations can include depth of field, illumination, and ergonomics. The exoscope has been introduced as an alternative to microscopes. In this report, we describe the application of the 3-dimensional (3D), high-definition (HD) exoscope for MIS-TLIF, and discuss its advantages and disadvantages.

\section{Methods}

Six patients were performed 3D-HD exoscope-assisted MIS-TLIF from September 2019 to December 2019. The exoscope was used [Kestrel View II, WEALTH CONCORD MITAKA MEDICAL TECHNOLOGIESCO., LTD. OF SHANGHAI, CHINA] during soft tissue dissection, discectomy, decompression, and cage insertion.

\section{Results}

The exoscope provided an excellent 3D view of the surgical field and allowed us to effectively and safely perform the surgical steps. All 6 patients experienced excellent surgical outcomes. The main advantages of the 3D exoscope include the possibility to provide excellent, magnified, and illuminated 3D-HD images of the surgical field to the entire operating room and serving as an excellent training tool.

\section{Conclusion}

We believe that the 3D exoscope will be a safe and effective tool to perform MIS-TLIF.

\section{Background}

Lumbar degenerative diseases including disc-herniation lumbar radiculopathy, lumbar spinal stenosis, lumbar spondylolisthesis, is one of the most common disorders in the lumbar spine region and often results in symptoms of lower back pain, leg pain, neurogenic claudication and decreased function. Prospective and randomized long-term data show that surgical intervention is available in whom conservative therapy fails to manage symptoms[1-4]. Surgical fusion has become one of the most commonly performed procedures in the treatment of lumbar degenerative diseases[5,6]. Transforaminal lumbar interbody fusion (TLIF) has been widely used in the surgical management of lumbar degenerative diseases[7,8]. Minimally invasive transforaminal lumbar interbody fusion (MIS-TLIF) aided with the tubule 
to finish decompression and interbody fusion is similar to open TLIF in clinical outcomes for the treatment of lumbar degenerative diseases[9.10]. Compared with open TLIF, MIS-TLIF has advantages of less blood loss, quicker recovery, and lower wound infection[8,11]. More recently, MIS-TLIF has become increasingly popular in the treatment of lumbar degenerative diseases.

The operating microscope with the advantages of significant magnification and illumination has played an important role in modern microsurgery when visualizing delicate vascular structures and spinal anatomy. Microscope assisted MIS-TLIF was reported favorable clinical outcomes and low complication rates[12-14]. However, although providing direct binocular and stereoscopic vision, limitations of the operating microscope can include depth of field, illumination, and ergonomics when working in deep corridors[15]. Furthermore, difficult maneuverability and encumbrance on the operative field are the major motivations for the limited use of a microscope in spinal procedures, particularly when dedicated surgical instruments need to be implanted[16]. To overcome these shortcomings, a novel visualization tool is required.

Recently, three dimensional (3D) exoscope, a new visualization tool with applications in some surgical disciplines, has been testified feasible in cadaver lab[17.18] and adopted in the spinal neurosurgery as an alternative to the microscope[19-23]. This exoscope is composed of a tubular microscope, a camera unit, a light source, a monitor, and a control unit. Compared with the traditional microscope, it has advantages such as improved ergonomics, an unblocked primary surgical field of vision, and a shared high-quality 3D view for the complete surgical team offering increased educational value[18:24].

We report our experience with a three-dimensional high-definition (3D-HD) exoscope system [Kestrel View II, WEALTH CONCORD MITAKA MEDICAL TECHNOLOGIESCO., LTD. OF SHANGHAI, CHINA] used as a visualization tool in 6 cases of MIS-TLIF. The surgical details, technical advantages, and limitations are included in this paper.

\section{Technical Report}

\section{Patient Population}

A total of 6 patients were performed MIS-TLIF by using the 3D exoscope, with an average age was $57.67 \pm 5.75$ years old. Operations were performed by the same surgeon, with resident assistance. The surgeon had prior experience using microscopic and endoscopic techniques for spinal applications. The detailed characteristic data were shown in Table 1. The 6 patients reported in this study had received information about the application of the 3D exoscope, which was included in the informed consent procedure for surgery.

\section{D Exoscopic Equipment}


The 3D-HD exoscope system [Kestrel View II, WEALTH CONCORD MITAKA MEDICAL TECHNOLOGIESCO. ,LTD. OF SHANGHAI, CHINA] is a visualization tool able to generate 3D real-time videos, which can be useful in several surgical disciplines. The system consists of a LED light source, a microscopic camera, and a HD screen (1920*1080 resolution), allowing for the generation of 3D videos. Optical/digital zooming results in magnification ranges from 1.9 to $39.3 x$. These devices are mounted on a mechanical arm. In the case of MIS-TLIF, surgeons wearing 3D glasses performed operation (figure 1).

\section{Surgical Procedures}

Patients underwent general anesthesia and placed as a prone position on the spine operating table. Then a $3 \mathrm{~cm}$ incision was made lateral to the midline on the decompression side. Through the incision, the tubular was inserted and fixed. The 3D exoscope was then positioned on the surgical sterile field. The camera orientation was adjusted to avoid any visual interference from the surgical instruments. The 3D exoscope was used during dissection, discectomy, decompression, and cage insertion. Surgeons wear special 3D glasses. The facet joints and the ligamentum flavum were removed by ultrasound scalpel and laminectomy rongeur. The nerve roots and the lateral edge of the dura were exposed and decompressed. The posterolateral annulus was incised, and discectomy was acted completely. A polyetheretherketone (PEEK) cage filled with autologous bone was inserted accurately. The homolateral pedicle screws were inserted after the cage implantation through the decompression incision. Posterior fixation on the contralateral side was done using percutaneous pedicle screws through two $1 \mathrm{~cm}$ incisions. A bent rod was placed to connect the pedicle screws. C-arm X-ray was used to make sure the position of the pedicle screws. Then, the drainage tube was placed underneath the fascia, and the wound was closed layer by layer. A typical case was shown in figure 2.

\section{Statistical Analysis}

All data were analyzed by using IBM SPSS Statistics (SPSS v23, IBM Corp., Armonk, NY, USA). The data measured at pre-operation, 1 week, 6 months after operation were compared by Wilcoxon rank-sum tests. A P-value $<0.05$ was considered significant in this study.

\section{Results}

The 3D exoscope offered excellent, magnified, and illuminated 3D-HD images of the surgical field in all the cases. All the cases were completed under 3D exoscope magnification from the points of view of the technique and the medicine. All patients had benefited from the procedures they underwent. All 6 patients experienced excellent surgical outcomes. VAS and ODI score of all the patients were demonstrated to decrease postoperatively $(P \otimes 0.05)$ (Table 2 ). The average operation time was $133.67 \pm 15.71 \mathrm{~min}$. The average blood loss was $91.67 \pm 17.51 \mathrm{ml}$ and the postoperative drainage was $60.00 \pm 13.78 \mathrm{ml}$. None of the 
operations had to be stopped because of technical problems. There was no conversion to the microscope or traditional open procedure in any operation. No complications occurred in this study.

\section{Discussion}

The operating microscope provides a significant intraoperative illumination and visualization of pathological tissue and anatomical structures, which makes the microneurosurgical procedure successful and safe. Thus, the microscope has become the mainstay instrument of neurosurgery[25]. However, there are some limitations in microscope including depth of field, illumination, and ergonomics when working in deep corridors[15]. Moreover, difficult maneuverability and encumbrance over the operative field are the motivations for the limited use of microscope during spinal procedures[16]. These limitations can influence the comfort of the surgeon and the surgical outcome[25]. Tools to improve these limitations are required.

It has been reported that the 3D exoscope has comparable results with the conventional operating microscope with good image quality and illumination during spinal surgery[22:23]. The 3D exoscope has a number of other advantages over the conventional operating microscope. Some studies reported the superiority of the exoscope compared with the operating microscope with regards to ergonomics and comfort[22'26]. The surgeons can straighten their back and even rest on the backrest in a heads-up position while performing surgery with exoscopes. The associated decrease of related exhaustion of ergonomics is important to preserving high-level surgeon performance over the course of a long case[27.28]. In addition, unlike the large frame and fixed bulky design of the conventional microscope, the other advantage of the 3D exoscope is related to its small size. The working distance is between 30 and $100 \mathrm{~cm}$, which provides unobstructed working space to the surgeons. As a result, this allows for comfortable use of the exoscope in the course of the MIS-TLIF procedure, such as neural structure decompression and cage insertion. Furthermore, Sack et al.[29] reported their experience using a 3D exoscope for microsurgery and concluded that with the shared surgical view, exoscopes possessed obvious educational advantages compared with the conventional microscope. Some investigators believed that exoscopes could be an alternative to the conventional microscope in some spinal procedures[20'23].

Recently, Ariffin et al.[19] reported the application of the 3D exoscope system as a visualization tool in spine surgery. They reported oblique lateral interbody fusion, MIS-TLIF, tubular decompression, and tubular microdiscectomy performed by using exoscopes, highlighting the advantages of the 3D exoscope in terms of visualization and ergonomics during spinal surgery. Nevertheless, they did not provide surgical details concerning the different steps of MIS-TLIF (dissection, discectomy, decompression, endplates preparation, cage insertion) assisted by 3D exoscopes. We used the novel 3D-HD exoscope as a visualization tool for minimally invasive transforaminal lumbar interbody fusion. The exoscope was used during dissection, discectomy, decompression, and cage insertion. In particular, neural structure decompression is a delicate surgical step and often requires the application of microsurgical techniques to obtain adequate magnification. In this study, all the patients experienced excellent surgical outcomes 
after effective decompression. VAS and ODI score of all the patients were demonstrated to decrease postoperatively. With the excellent 3D surgical field provided by the exoscope system, it could help reduce the risk of nerve injury. No complications occurred in this study. Furthermore, surgeons can clearly identify the bleeding vessels which are conducive to the timely and accurate electric coagulation.

One of the main advantages of the 3D exoscope compared with the conventional microscope is the possibility to provide a 3D surgical field to the entire operating room. From learning perspectives, it makes more sense that the assistant observes the same view used by the primary surgeon intraoperatively. Therefore, it can facilitate resident and fellow education in complicated procedures. Some investigators also believed that the 3D exoscope served as an excellent teaching tool[22:29].

As already reported by the majority of authors, the one major disadvantage of the exoscope was the cumbersomeness in repositioning and refocusing[23.30]. This inconvenience is attributed to the mechanical holding arm. During surgical procedures, surgeons often need to refocus and reposition the scope without losing sight or moving hands off the surgical field. However, the exoscope system needs surgeons to move the mechanical arm to finish the repositioning and refocusing for getting a clear surgical field in different steps of the operation. It obliges the surgeon to stop the operation and move hands away from the operating table. Too many interruptions can limit the fluidity of surgery, increasing operation time. In addition, microsurgery means continuous use of both hands and in case of brisk bleeding having to adjust the instrument by hand might be extremely dangerous. Therefore, Rossini et al. [30] suggests the development of a footplate command mandatory for safe clinical utilization of the exoscope. Besides, although most neurosurgeons today are accustomed to an operative microscope, there is certainly a learning curve necessary before using the exoscope in spinal surgery. Belykh et al.[26] reported a significant learning curve exists for becoming accustomed to operating with the exoscope system. No doubt it could be a potential issue for operators who are not familiar with the comparable microscope setup. The surgeons involved in the study had previous experience utilizing the microscope and only had a short learning curve when performing the surgery with a 3D microscope.

\section{Conclusion}

The exoscope is a visualization tool gradually entering the microsurgical practice. Currently, few cases have been approached with this instrument. The main disadvantage highlighted by previous reports is the cumbersomeness in repositioning and refocusing. The advantages of the 3D exoscope include providing the excellent, magnified, and illuminated 3D-HD images of the surgical field and serving as an excellent training tool. We believe that 3D exoscopes will be a safe and effective tool to perform MIS-TLIF.

\section{Abbreviations}

MIS-TLIF: minimally invasive transforaminal lumbar interbody fusion

3D: three-dimensional 
HD: high-definition

TLIF: transforaminal lumbar interbody fusion

\section{References}

1. Weinstein JN, Lurie JD, Tosteson TD, Zhao W, Blood EA,Tosteson AN, et al. Surgical compared with nonoperative treatment for lumbar degenerative spondylolisthesis. four-year results in the Spine Patient Outcomes Research Trial (SPORT) randomized and observational cohorts. J Bone Joint Surg Am 2009; 91(6): 1295-1304.

2. Weinstein JN, Lurie JD, Tosteson TD, Tosteson AN, Blood EA,Abdu WA, et al. Surgical versus nonoperative treatment for lumbar disc herniation: four-year results for the Spine Patient Outcomes Research Trial (SPORT). Spine (Phila Pa 1976) 2008; 33(25): 2789-2800.

3. Weinstein JN, Tosteson TD, Lurie JD, Tosteson A, Blood E,Herkowitz H, et al. Surgical versus nonoperative treatment for lumbar spinal stenosis four-year results of the Spine Patient Outcomes Research Trial. Spine (Phila Pa 1976) 2010; 35(14): 1329-1338.

4. Shamji MF, Mroz T, Hsu W,Chutkan N. Management of Degenerative Lumbar Spinal Stenosis in the Elderly. Neurosurgery 2015; 77 Suppl 4(S68-S74.

5. Yoshihara H,Yoneoka D. National trends in the surgical treatment for lumbar degenerative disc disease: United States, 2000 to 2009. Spine J 2015; 15(2): 265-271.

6. Yavin D, Casha S, Wiebe S, Feasby TE, Clark C,Isaacs A, et al. Lumbar Fusion for Degenerative Disease: A Systematic Review and Meta-Analysis. Neurosurgery 2017; 80(5): 701-715.

7. Lan T, Hu SY, Zhang YT, Zheng YC, Zhang R,Shen Z, et al. Comparison Between Posterior Lumbar Interbody Fusion and Transforaminal Lumbar Interbody Fusion for the Treatment of Lumbar Degenerative Diseases: A Systematic Review and Meta-Analysis. World Neurosurg 2018; 112(86-93.

8. Phan K, Rao PJ, Kam AC,Mobbs RJ. Minimally invasive versus open transforaminal lumbar interbody fusion for treatment of degenerative lumbar disease: systematic review and meta-analysis. Eur Spine J 2015; 24(5): 1017-1030.

9. Goldstein CL, Phillips FM,Rampersaud YR. Comparative Effectiveness and Economic Evaluations of Open Versus Minimally Invasive Posterior or Transforaminal Lumbar Interbody Fusion: A Systematic Review. Spine (Phila Pa 1976) 2016; 41 Suppl 8(S74-S89.

10. Hammad A, Wirries A, Ardeshiri A, Nikiforov O,Geiger F. Open versus minimally invasive TLIF: literature review and meta-analysis. J Orthop Surg Res 2019; 14(1): 229.

11. Hari A, Krishna M, Rajagandhi S,Rajakumar DV. Minimally invasive transforaminal lumbar interbody fusion-indications and clinical experience. Neurol India 2016; 64(3): 444-454.

12. Peng $P$, Chen $K$, Chen H, Zhang K, Sun J,Yang P, et al. Comparison of O-arm navigation and microscope-assisted minimally invasive transforaminal lumbar interbody fusion and conventional transforaminal lumbar interbody fusion for the treatment of lumbar isthmic spondylolisthesis. $J$ Orthop Translat 2020; 20(107-112. 
13. Chen K, Chen H, Zhang K, Yang P, Sun J,Mo J, et al. O-arm Navigation Combined With Microscopeassisted MIS-TLIF in the Treatment of Lumbar Degenerative Disease. Clin Spine Surg 2019; 32(5): E235-E240.

14. Zhang W, Li X, Shang X, Xu X, Hu Y,He R, et al. Modified minimally invasive transforaminal lumbar interbody fusion using a trans-multifidus approach: a safe and effective alternative to open-TLIF. $J$ Orthop Surg Res 2015; 10(93.

15. Bakhsheshian J, Strickland BA, Jackson C, Chaichana KL, Young R,Pradilla G, et al. Multicenter Investigation of Channel-Based Subcortical Trans-Sulcal Exoscopic Resection of Metastatic Brain Tumors: A Retrospective Case Series. Oper Neurosurg (Hagerstown) 2019; 16(2): 159-166.

16. Adogwa O, Elsamadicy A, Reiser E, Ziegler C, Freischlag K,Cheng J, et al. Comparison of surgical outcomes after anterior cervical discectomy and fusion: does the intra-operative use of a microscope improve surgical outcomes. J Spine Surg 2016; 2(1): 25-30.

17. Moisi MD, Hoang K, Tubbs RS, Page J, Fisahn C,Paulson D, et al. Advancement of Surgical Visualization Methods: Comparison Study Between Traditional Microscopic Surgery and a Novel Robotic Optoelectronic Visualization Tool for Spinal Surgery. World Neurosurg 2017; 98(273-277.

18. Herlan S, Marquardt JS, Hirt B, Tatagiba M,Ebner FH. 3D Exoscope System in NeurosurgeryComparison of a Standard Operating Microscope With a New 3D Exoscope in the Cadaver Lab. Oper Neurosurg (Hagerstown) 2019; 17(5): 518-524.

19. Ariffin M, Ibrahim K, Baharudin A,Tamil AM. Early Experience, Setup, Learning Curve, Benefits, and Complications Associated with Exoscope and Three-Dimensional 4K Hybrid Digital Visualizations in Minimally Invasive Spine Surgery. Asian Spine J 2020; 14(1): 59-65.

20. Barbagallo G,Certo F. Three-Dimensional, High-Definition Exoscopic Anterior Cervical Discectomy and Fusion: A Valid Alternative to Microscope-Assisted Surgery. World Neurosurg 2019; 130(e244-e250.

21. Kwan K, Schneider JR, Du V, Falting L, Boockvar JA,Oren J, et al. Lessons Learned Using a HighDefinition 3-Dimensional Exoscope for Spinal Surgery. Oper Neurosurg (Hagerstown) 2019; 16(5): 619-625.

22. Oertel JM,Burkhardt BW. Vitom-3D for Exoscopic Neurosurgery: Initial Experience in Cranial and Spinal Procedures. World Neurosurg 2017; 105(153-162.

23. Krishnan KG, Schöller K,Uhl E. Application of a Compact High-Definition Exoscope for Illumination and Magnification in High-Precision Surgical Procedures. World Neurosurg 2017; 97(652-660.

24. Roethe AL, Landgraf P, Schröder T, Misch M, Vajkoczy P,Picht T. Monitor-based exoscopic 3D4k neurosurgical interventions: a two-phase prospective-randomized clinical evaluation of a novel hybrid device. Acta Neurochir (Wien) 2020.

25. Muhammad S, Lehecka M,Niemelä M. Preliminary experience with a digital robotic exoscope in cranial and spinal surgery: a review of the Synaptive Modus V system. Acta Neurochir (Wien) 2019; 161(10): 2175-2180.

26. Belykh EG, Zhao X, Cavallo C, Bohl MA, Yagmurlu K,Aklinski JL, et al. Laboratory Evaluation of a Robotic Operative Microscope - Visualization Platform for Neurosurgery. Cureus 2018; 10(7): e3072. 
27. Fargen KM, Turner RD,Spiotta AM. Factors That Affect Physiologic Tremor and Dexterity During Surgery: A Primer for Neurosurgeons. World Neurosurg 2016; 86(384-389.

28. Berguer R. Surgery and ergonomics. Arch Surg 1999; 134(9): 1011-1016.

29. Sack J, Steinberg JA, Rennert RC, Hatefi D, Pannell JS,Levy M, et al. Initial Experience Using a HighDefinition 3-Dimensional Exoscope System for Microneurosurgery. Oper Neurosurg (Hagerstown) 2018; 14(4): 395-401.

30. Rossini Z, Cardia A, Milani D, Lasio GB, Fornari M,D'Angelo V. VITOM 3D: Preliminary Experience in Cranial Surgery. World Neurosurg 2017; 107(663-668.

\section{Declarations}

\section{Ethics approval and consent to participate}

All patients reported in this study had received information about the application of the 3D exoscope, which was included in the informed consent procedure for surgery.

\section{Consent for publication}

Written informed consent for publication of their clinical details and/or clinical images was obtained from the patient.

\section{Availability of data and materials}

All the data of the manuscript are presented in the paper.

\section{Competing interests}

We have no conflict of interest which may influence the outcomes of this study.

\section{Funding}

This work was supported by the Natural Science Foundation of China (Grantno:81401802); and the Natural Science Foundation of Hubei Province (Grant no:2018CFB487).

\section{Tables}


Table 1 The demographic data of the patients

\begin{tabular}{|c|c|c|c|c|c|c|c|}
\hline ID & Gender & Age(years) & Diagnosis & Level & $\begin{array}{l}\text { Operation } \\
\text { time }(\mathrm{ml})\end{array}$ & $\begin{array}{l}\text { Blood } \\
\text { loss }(\mathrm{ml})\end{array}$ & $\begin{array}{l}\text { Postoperative } \\
\text { drainage }(\mathrm{ml})\end{array}$ \\
\hline 1 & male & 48 & Spondylolisthesis & $\begin{array}{l}\text { L5- } \\
\text { S1 }\end{array}$ & 132 & 80 & 50 \\
\hline 2 & male & 55 & Disk herniation & $\begin{array}{l}\text { L4- } \\
\text { L5 }\end{array}$ & 115 & 65 & 45 \\
\hline 3 & female & 62 & Disk herniation & $\begin{array}{l}\text { L4- } \\
\text { L5 }\end{array}$ & 124 & 100 & 65 \\
\hline 4 & male & 57 & Spinal stenosis & $\begin{array}{l}\text { L5- } \\
\text { S1 }\end{array}$ & 146 & 115 & 80 \\
\hline 5 & female & 60 & Spondylolisthesis & $\begin{array}{l}\text { L4- } \\
\text { L5 }\end{array}$ & 158 & 100 & 50 \\
\hline 6 & female & 64 & Disk herniation & $\begin{array}{l}\text { L5- } \\
\text { S1 }\end{array}$ & 127 & 90 & 70 \\
\hline
\end{tabular}

Table 2 Postoperative VAS and ODI scores of the patients

\begin{tabular}{llll}
\hline & VAS-back pain & VAS-leg pain & ODI \\
\hline preoperative & $6.22+1.06$ & $6.83+0.80$ & $59.67+8.48$ \\
1 week after operation & $2.53+0.65^{*}$ & $2.23+0.52^{*}$ & $27.67+4.18^{*}$ \\
6 months after operation & $1.87+0.62^{*}$ & $1.95+0.50^{*}$ & $18.00+3.63^{*}$ \\
\hline $\begin{array}{l}\text { VAS = visual analogue scale; ODI= Oswestry disability } \\
\text { pre-operative data }\end{array}$ & \multicolumn{3}{l}{${ }^{*}, P<0.05$ compared to the }
\end{tabular}

\section{Figures}




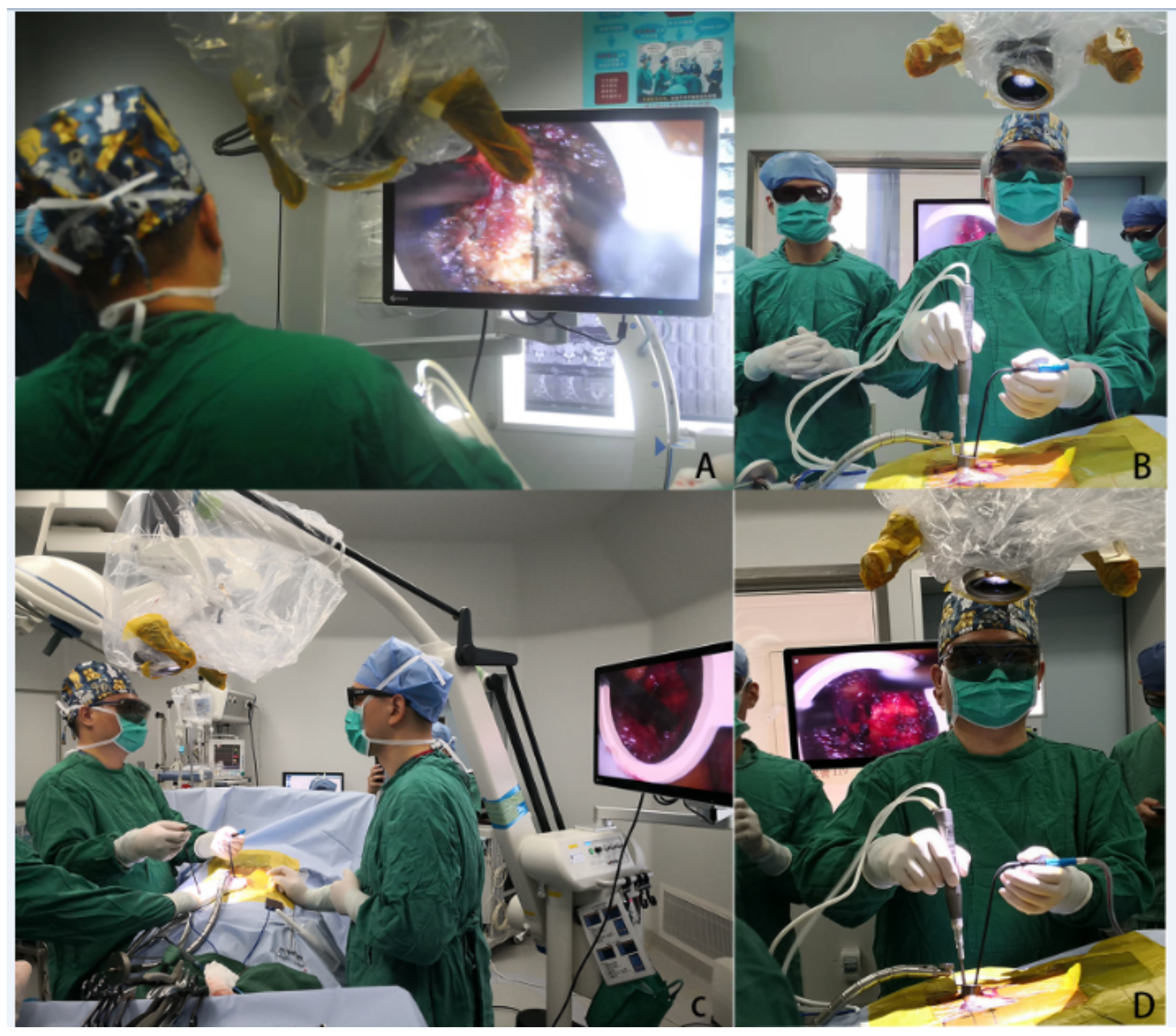

Figure 1

(A-D) Surgeons performed MIS-TLIF with a 3D exoscope. 

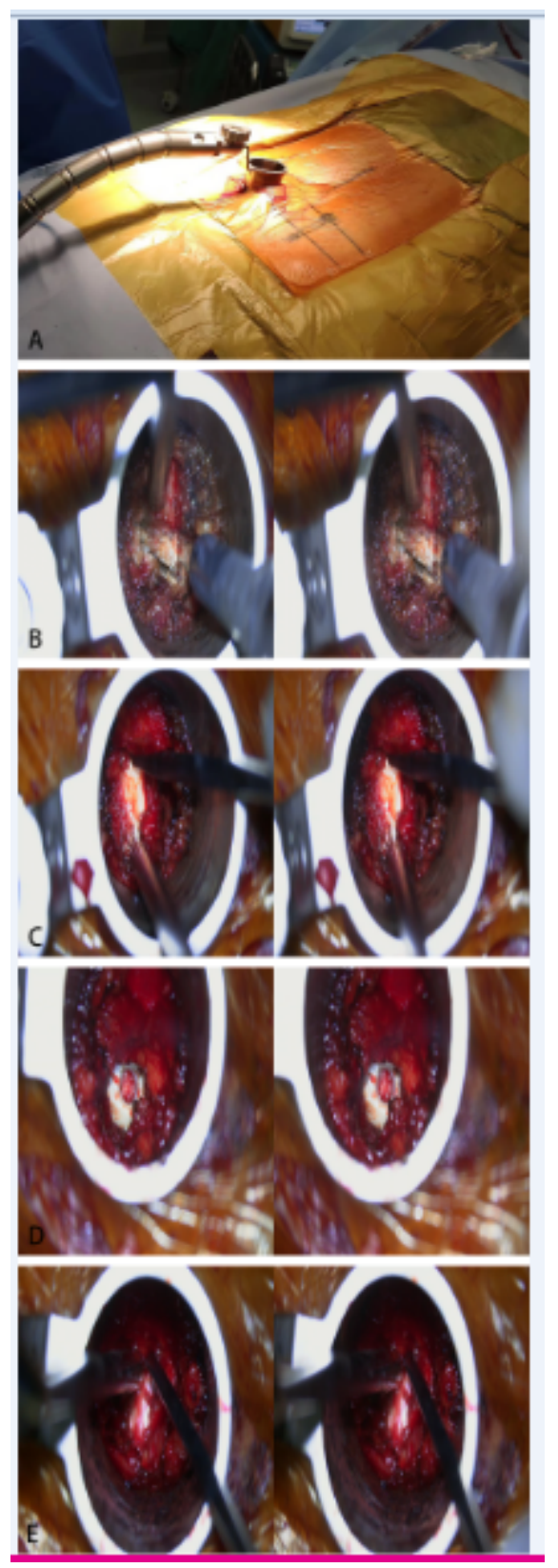

Figure 2

A, The tubular was inserted and fixed through a $3 \mathrm{~cm}$ incision. B, Ultrasound scalpel was used to finish laminectomy. C, The Annulus fibrosus was exposed clearly. D, A cage filled with autologous cancellous bone was inserted into the disc space; E, The nerve root was decompressed completely. 